\title{
Une lecture de Comment j'ai vidé la maison de mes parents de Lydia Flem
}

Pour une approche matérielle du deuil

A Reading of Comment j'ai vidé la maison de mes parents by Lydia Flem. For

a Material Approach of Bereavement

Dominique Carlat

\section{CpenEdition}

Journals

Édition électronique

URL : https://journals.openedition.org/recherchestravaux/3112

DOI : 10.4000/recherchestravaux.3112

ISSN : 1969-6434

Éditeur

UGA Éditions/Université Grenoble Alpes

Édition imprimée

ISBN : 978-2-37747-241-3

ISSN : 0151-1874

Référence électronique

Dominique Carlat, « Une lecture de Comment j'ai vidé la maison de mes parents de Lydia Flem »,

Recherches \& Travaux [En ligne], 97 | 2020, mis en ligne le 12 novembre 2020, consulté le 29 juin 2021

URL : http://journals.openedition.org/recherchestravaux/3112 ; DOI : https://doi.org/10.4000/ recherchestravaux.3112

Ce document a été généré automatiquement le 29 juin 2021.

(c) Recherches \& Travaux 


\title{
Une lecture de Comment j'ai vidé la maison de mes parents de Lydia Flem
}

Pour une approche matérielle du deuil

\author{
A Reading of Comment j'ai vidé la maison de mes parents by Lydia Flem. For \\ a Material Approach of Bereavement
}

Dominique Carlat

1 Psychanalyste, biographe de Freud, écrivaine ayant proposé une approche historique et sociologique de l'invention de la psychanalyse dans la Vienne cosmopolite de l'empire des Habsbourg ${ }^{1}$, Lydia Flem structure son récit Comment j'ai vidé la maison de mes parents ${ }^{2}$ autour d'éléments théoriques empruntés au père de la psychanalyse. Les deux épigraphes, extraites l'une de la correspondance de Freud (lettre à Max Eitington, $1^{\text {er }}$ décembre 1929), l'autre de l'un de ses premiers essais (Traumdeutung, 1908), revêtent une double fonction : faire allégeance à celui dont on revendique l'héritage théorique et évoquer, à travers l'exemple du fondateur, le bénéfice que l'on souhaite tirer d'une telle expérience d'écriture: l'avancée dans une forme d'auto-analyse usant du lectorat imaginé comme d'un pôle transférentiel. Après la mort des deux parents (sa mère vient de mourir, deux ans après son père), le deuil ne s'accomplirait qu'à travers le passage de cette étape décisive : la confrontation à l'univers qu'ils habitaient, l'acceptation de la perte irrémédiable de ce dernier, territoire jusque-là propice aux identifications narcissiques. Le «vide» à creuser au milieu de la multitude d'objets "laissés orphelins " est ainsi présenté comme un processus nécessaire, indispensable à la réalisation du deuil. Ce travail est d'autant plus nécessaire que la transmission s'est faite par convention, sans explicitation, et qu'elle submerge la descendante sous les mille témoignages d'existences désormais arrêtées. La charge affective de ce travail est très lourde, notamment parce que Lydia Flem découvre les témoignages d'une histoire dont ses parents ont voulu lui épargner le poids : celle de rescapés de la Shoah.

2 Cependant, par son titre et le prosaïsme qu'il affiche, ce livre dépasse cette dimension testimoniale, réflexive et illustrative ; il atténue par son pragmatisme revendiqué les effets pathétiques de l'arrière-fond historique. «Comment j'ai vidé la maison de mes 
parents », cela ne signifie pas seulement "Comment diable suis-je parvenue, malgré l'aspect sisyphéen de la tâche, à vider cette maison, à faire mien cet héritage ?» (et le diable est peut-être effectivement de la partie, qui fait encore réapparaître des objets et des pièces quand on croyait avoir bientôt achevé...). C'est aussi, livré au lecteur, adressé à un public, un petit traité méthodique, un témoignage à dimension exemplaire : je peux, selon une approche anglo-saxonne, évoquer pour vous les étapes par lesquelles je suis passée dans cette entreprise - banale, commune et titanesque - qui a consisté à vider ce lieu unique - unique par la charge affective qu'il recouvre, l'investissement narcissique auquel depuis des années il a donné lieu.

3 Exposer au lectorat mes " méthodes " pour réaliser cette tâche ingrate liée à l'héritage, cela permet de lever partiellement le refoulement qu'a suscité l'appropriation des biens parentaux, de partager avec autrui la répugnance qu'il m'a fallu vaincre. Et si cela relève d'un interdit difficile à braver (comment se peut-il que j'aie soudain la jouissance de ce qui, il y a peu, ne m'appartenait pas ?), c'est, note lucidement l'écrivaine, parce que c'est la loi qui me fait dépositaire de ces biens, et non pas une volonté expressément édictée par testament. Le terme dépôt suggère des connotations que le texte va déployer : en rangeant la maison, chacune de ses pièces, c'est en effet toute l'intimité accumulée depuis des années, des décennies, que l'héritière se trouve contrainte de déranger, au risque de brouiller sa perception et sa représentation du temps, de l'espace et de son identité ; comme d'une eau dont les alluvions jusqu'ici déposées en son fond viendraient à oblitérer la transparence...

4 Mais d'être ainsi exposée et transmise au lecteur, l'expérience se fait-elle moins sacrilège, elle qui nécessite d'aller fouiller - bien malgré soi - dans l'intimité du père et de la mère, de résoudre à leur place la question de la sauvegarde ou de la destruction ? Sacrilège avoué et partagé serait-il à demi pardonné ? Le don de la recette pour réussir à vider cet héritage participe indubitablement de la même générosité qui régissait l'entreprise de divulgation et de popularisation de la psychanalyse ${ }^{3}$. J'ai parlé de "méthode » à propos de ce travail qui incombe à l'enfant survivant à la mort des parents. Et c'est en effet ce que le titre retenu semble présupposer. Accomplir ce devoir, c'est se savoir désormais " sans personne derrière soi » : définitivement arraché au temps de l'enfance. Cependant la narratrice nous livre-t-elle le récit maîtrisé et linéaire d'une démarche rationnelle, fondée sur des principes, des règles, le franchissement d'étapes permettant d'accéder à un résultat satisfaisant? Loin de s'ériger en détentrice d'une telle connaissance surplombante - ou d'un savoir résumable -, l'écrivaine, à travers l'organisation de ses chapitres et l'adoption de tempos différents, nous invite à accepter le rythme irrationnel qu'adopte immanquablement cette expérience matérielle qui accompagne le deuil. Lydia Flem associe le rythme irrégulier - fait de stases puis de déchaînements - à celui du « flot bouillonnant des affects » (p. 149). Entre douceur et agressivité, peine et rage, nostalgie et colère rétrospective, "tourments et retournements" (p.150) se succèdent, s'affrontent et se neutralisent un instant, avant de se redéployer. Car c'est bien de la douleur qu'il faut s'approcher, pour tenter de la conjurer. La répétition règne en maître, associée ici à un imaginaire du souffle et de ses irrégularités : halètement, asphyxie, profonde inspiration...

5 Mais la psyché n'est pas seule en cause, ni l'inconscient corporel: le monde matériel laissé par les parents, avec ses milliers d'objets imposant leur présence et leur opacité, devient un lieu d'exploration, d'aventure spirituelle mais aussi de résistance. Il oppose 
son silence, sa cohérence absurde, son désordre névrotique, son insignifiance. Aussi se prête-t-il à la rêverie, mais parfois aussi à des lectures fantasmatiques qu'autrui n'est plus là pour interrompre. Ce monde constitue une masse indifférenciée, un nombre incalculable d'objets dont il va falloir choisir le destin : les retirer de la circulation ou les relancer dans le jeu ininterrompu de la vie ? D'où le découragement qui vous gagne, le sentiment d'une tâche démesurée que jamais l'on ne parviendrait à épuiser et dont on hésite à porter la responsabilité. En effet, devenir orphelin, y compris à travers ce passage initiatique, c'est probablement être seul désormais à pouvoir répondre. Le récit de Lydia Flem trouve son rythme spécifique dans cette reprise permanente, ce désarroi sans cesse combattu et renaissant: alors qu'une étape vient d'être franchie, que des pièces ont enfin été vidées, ressurgit brusquement un espace comble, un meuble bourré à craquer, une alcôve dissimulant un univers en réduction... Il n'y va pas de magie noire; juste de la révélation matérielle d'une loi psychologique : les morts nous ont laissé une myriade de signes dont nous avons souvent le sentiment de n'être ni les destinataires, ni les bons interprètes. Nous avons beau les scruter, les classifier, les interroger, très peu se plient à notre volonté et accèdent à un sens transparent : un reste nous échappe. Mais ce reste est aussi ce qui nous conduit au cœur du présent, de nos désirs.

6 C'est pourquoi à l'instant même où nous sommes littéralement débordés, enfouis sous l'ampleur du legs, se fait sentir un vide, impossible à combler puisque la parole est désormais interdite: "le chagrin se creuse. Avec des moments de vide, d'absence, de tumulte» (p. 151). Une oscillation permanente nous fait passer de l'excès au manque, de la béance au fouillis, de la désespérance à une singulière qualité de ferveur. À travers l'attitude affective négociée avec les objets laissés par les parents disparus se conquiert ainsi peu à peu, fragilement (avec ses avancées et ses ressauts), une distance moins instable - plus supportable - à l'égard de nos morts. Lydia Flem qualifie ce processus du juste terme de "remaniement »: «ce sont d'intenses moments de remaniements intérieurs " (p.150). L'ordre peu à peu conquis, s'il recouvre donc une dimension métaphorique, ne doit pas cependant être coupé de sa dimension matérielle: la facticité des objets retenus et "archivés » en fait des documents, des traces toujours disponibles désormais pour construire une mémoire libre, dégagée autant que possible de toute part fantasmatique, préservée de toute projection sur les morts, ou identification à leurs figures. «Les documents que je grappillai en divers lieux de la maison établissaient les faits, crus mais clairs et distincts, sans l'ombre d'une émotion, sans le risque d'une fusion mortifere»" (p. 75, je souligne). Mais avec cette vision rationnelle, idéale, presque clinique (" établir les faits »), ne touche-t-on pas la limite de l'homologie fondatrice du texte? Maîtriser peu à peu l'espace domestique des parents, ce chaos arrêté au moment de leur mort, y choisir des « documents » que l'on garde, en jeter les traces jugées anecdotiques, est-ce vraiment effectuer un travail qui permettrait de maîtriser provisoirement l'inconscient, de dégager un récit épargné par le fantasme ? Peut-être est-ce davantage réaliser un tour de force, arracher à ce théâtre abandonné par les prédécesseurs les accessoires de sa propre scène intime. Et en effet, ranger la maison parentale avant de la céder, "y faire place nette " selon la lucide expression, permet d'éprouver l'ambivalence des sentiments qui m'ont lié aux parents. Mais il y a aussi chez Lydia Flem un optimisme de principe qui veut ici qu'avec la fin de cette tâche titanesque le travail inconscient trouve, sinon son achèvement, du moins une trêve : que l'ordonnancement opéré (avec le transfert des objets conservés dans sa propre demeure ou leur don à des amis) ouvre sur une mémoire apaisée, ouverte à la 
vie. Reconnaître la violence nécessaire pour briser les entraves qui nous relient au passé, cela suffit-il pour s'en défaire radicalement, définitivement? C'est le pari qu'entend relever le récit, retrouvant dans cette confiance en la vie le legs laissé par les survivants de la Shoah : avec le traumatisme, ravauder l'existence, maintenir l'élégance d'une posture, la vitalité d'un geste.

7 La dimension ouvertement sacrilège de la fouille opérée dans les affaires des défunts (évoquée p. 49 ou p. 87) est alors elle-même représentée comme un moyen de lutter contre la tentation mortifère que François Truffaut a illustrée dans La Chambre verte: celle de transformer la demeure en musée rempli de multiples autels voués au culte des morts. Pour autant, l'héritière, enfant unique, sait qu'elle ne sera jamais quitte. Aussi Lydia Flem recourt-elle à l'image d'une dette imprescriptible : « même après la mort de nos parents, ne cessons-nous jamais de vivre pour eux, à travers eux, en fonction d'eux et contre eux ? Est-ce une dette qui nous poursuit toujours? » (p. 89)

8 Le souvenir de cette question fait que le soulagement exprimé à la fin du récit revêt une valeur ambiguë: certes, domine le contentement d'avoir enfin accompli la tâche gigantesque, démesurée que souvent l'on a crue au-dessus de nos forces. Cette joie est associée à l'affirmation d'une juste distance enfin gagnée à l'égard des deux figures parentales : "Oserais-je le confier à ces pages? En me quittant, ils (mes parents) me libéraient de leur emprise muette. Ils étaient morts. J'allais enfin pouvoir les rencontrer» (p.96). Et à travers la description des objets triés et restitués à la circulation de la vie, le récit semble en effet souvent s'efforcer de convertir ce qui dans le mot «orphelin » rappelle la privation et le vide en témoignage d'une richesse, d'une plénitude conquise : état digne d'un âge d'or ${ }^{4} . .$. La perte est aussi libération. Pourtant là où une maison parentale peut se vider, là où cette obligation qui vous incombe socialement peut être peu à peu accomplie - non sans efforts -, la scène intime demeure ouverte à de nouveaux bouleversements, à d'autres recompositions, à d'autres apparitions spectrales : ni la honte, ni la culpabilité - à travers le remords - ne se dissolvent aussi magiquement.

Comment j'ai vidé la maison de mes parents, jusque dans l'aspect accompli du passé composé utilisé, constitue donc un récit qui souhaite projeter sur un temps en train de s'achever (grâce au présent de l'écriture) cette nécessité où l'on s'est trouvé de "faire avec " les signes laissés par les parents morts. Certes, le texte évoque la dimension sacrilège de ce tri opéré dans les traces qu'ils ont laissées. Mais c'est pour mieux construire de soi une image héroïque : celle d'une enfant qui a conquis le droit de saisir la joie, qui a trouvé dans le bric-à-brac effrayant et merveilleux du passé de quoi enchanter, malgré tout, son monde ${ }^{5}$. C'est pourquoi je proposerais volontiers d'associer cette "auto-fiction familiale " à l'activité photographique à laquelle Lydia Flem va s'adonner dans les années suivantes : ici et là il s'agit par l'imaginaire de tenter de résoudre les failles symboliques, de recoudre les déchirures de la transmission: d'accomplir magiquement une réparation. Il en ira de même dans le texte romanesque La Reine Alice, où le récit autobiographique du traitement par chimiothérapie du cancer est rendu possible par le recours à l'univers fictionnel, merveilleux, d'Alice au pays des merveilles. Lewis Caroll, photographe et rêveur, ouvre la voie vers ces paysages intérieurs « soulignant l'irréalité du réel, la réalité de l'irréel ${ }^{6}$ ». 


\section{NOTES}

1. L. Flem, La Vie quotidienne de Freud et de ses patients, Paris, Hachette, 1986.

2. L. Flem, Comment j'ai vidé la maison de mes parents, Paris, Seuil, coll. « La librairie du Xxi ${ }^{\mathrm{e}}$ siècle », 2004. Les références à cette œuvre seront données entre parenthèses.

3. L. Flem, L'Homme Freud, Paris, Seuil, coll. « La librairie du XxI siècle », 1991.

4. Lydia Flem évoque ici la découverte de la correspondance amoureuse de ses parents : celle-là même qui donnera naissance en 2006, dans la même collection, aux Lettres d'amour en héritage.

5. Sous une de ses photographies, Lydia Flem notera cette pensée de Nietzsche : "Je suis préparé au pire, confiant néanmoins que de l'excès de souffrance et de peur naîtra ici et là la fleur nocturne de la connaissance" (Journal implicite, La Martinière/Musée Européen de la photographie, 2013).

6. S. Monjour, "Lydia Flem au miroir de Claude Cahun: une poétique de l'anamorphose ", communication au colloque "Héritages de Claude Cahun et Marcel Moore", organisé par Andrea Oberhuber et Alexandra Arvisais, 28 et 29 mai 2015, université de Montréal. On se reportera également au « Portrait de Lydia Flem » publié par Claude Burgelin dans le numéro 33 de la revue Les Moments littéraires, 2015.

\section{RÉSUMÉS}

Avec Comment j'ai vidé la maison de mes parents, Lydia Flem nous offre une réflexion sur la manière dont le deuil est aussi un ensemble de gestes concrets qui lui donnent une matérialité. En vidant la maison de ses parents décédés, l'auteure se plonge dans leur passé en inspectant la multitude d'objets qui les entouraient. Mais ce tri, nécessairement sacrilège, participe aussi du travail de deuil que l'écrivaine psychanalyse place sous le patronage de Freud.

With Comment j'ai vidé la maison de mes parents, Lydia Flem questions the way in which mourning is also a set of concrete gestures that give it materiality. By emptying the house of her dead parents, the author delves into their past by inspecting the multitude of objects that surrounded them. But this selection, necessarily sacrilegious, is also part of the work of mourning that the psychoanalytical writer places under Freud's patronage.

\section{INDEX}

Mots-clés : Psychanalyse, deuil, objets, répétition

Keywords : Psychoanalysis, bereavement, objects, repetition 


\section{AUTEUR}

\section{DOMINIQUE CARLAT}

Université Lyon 2

Dominique Carlat est professeur de Littérature française moderne et contemporaine à l'université Lyon 2. Il est notamment l'auteur, aux éditions José Corti, de Ghérasim Luca, l'intempestif (1998) et de Témoins de l'inactuel (2007), un essai consacré au deuil chez Claude Esteban, Michel Deguy, Roland Barthes et Pierre Pachet. 